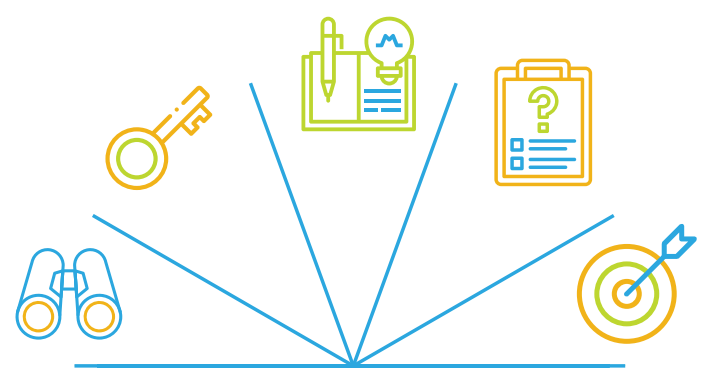

\title{
PESOUISA-AÇÃO NO CONTEXTO DO PROJETO RESPOSTA RÁPIDA À SÍFILIS: EXPERIÊNCIA DO USO DA METODOLOGIA DA PROBLEMATIZAÇÃO COM O ARCO DE MAGUEREZ
}

ACTION RESEARCH IN CONTEXT OF THE PROJECT QUICK RESPONSE TO SYPHILIS:

EXPERIENCE WITH USING OF THE PROBLEMATIZATION METHODOLOGY WITH

MAGUEREZ'S ARCH

\section{Paula Guidone Pereira Sobreira}

Mestre em Saúde Pública (ENSP/FIOCRUZ). Pós-graduação lato sensu em Psicoterapia Infanto Juvenil (IFF/FIOCRUZ). Psicóloga pela Universidade Gama Filho. Apoiadora do Ministério da Saúde/UFRN no Projeto de Resposta Rápida à Sífilis nas Redes de Atenção. Professora e coordenadora de Pós-graduação e Extensão da UNIG. e-mail:paulaguidone@yahoo.com.br

Adriano Santiago Dias dos Santos

Gestor de Serviços de Saúde pela Universidade Federal de Minas Gerais - UFMG, Mestrando em Saúde

Coletiva pela Universidade de Brasília - UnB, Consultor do Departamento de Doenças de Condições Crônicas e Infecções Sexualmente Transmissíveis atuando na Coordenação Geral de Vigilância às Infecções Sexualmente Transmissíveis - DCCI/SVS/MS e-mail: mscadriano@gmail.com

\section{Lutigardes Bastos Santana}

Odontóloga pela Universidade Federal da Bahia, Especialista em Saúde Coletiva e Sociedade pelo Centro Universitário Internacional e em Saúde da Família pela UFBA, Consultora do Departamento de Doenças de Condições Crônicas e Infecções Sexualmente Transmissíveis atuando na Coordenação Geral de Vigilância às Infecções Sexualmente Transmissíveis - DCCI/SVS/MS - e-mail: lutisantana@hotmail.com. Especialista em Gestão do Trabalho e Educação em Saúde pela Universidade Federal do Rio Grande do Norte (UFRN). Enfermeira do Complexo Hospital de Clínicas do Paraná (CHC-UFPR). E-mail: gil-mara-dasilva@hotmail.com

Mario Jorge Sobreira da Silva

Doutor e Mestre em Saúde Pública pela Escola Nacional de Saúde Pública (ENSP/FIOCRUZ). Graduação em Farmácia. Chefe da Divisão de Ensino no Instituto Nacional do Câncer (INCA). e-mail: mjsobreira@yahoo.com.br

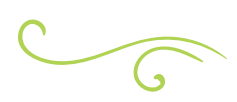

\section{RESUMO}

O objetivo deste artigo é relatar a experiência da realização de uma oficina junto aos profissionais de saúde, visando ao planejamento de ações voltadas para o combate à sífilis. Tomando como base a metodologia da pesquisa-ação, ocorreu uma oficina em uma Clínica da Família localizada em um município da Baixada Fluminense/ RJ. $O$ estudo empregou a metodologia da problematização, tendo como referência a Teoria do Arco de Charles Maguerez 
analisando o funcionamento cíclico em 5 etapas: observação da realidade; definição dos pontos-chave; teorização; proposição de hipóteses de solução; e, aplicação à realidade. A análise dos dados observou cada uma das etapas, com relatório produzido sobre a experiência. Como resultado, o estudo apresentou oito propostas para - alcance dos objetivos do Projeto de Resposta Rápida à Sífilis. A vivência dessa prática permitiu desenvolver um processo de ação-reflexão-ação das atividades assistenciais exercidas pelos profissionais de saúde da unidade em questão, sendo um exemplo de trabalho efetivo do apoiador em conjunto com os atores do território visando produzir mudanças nas práticas de gestão e assistência na busca de melhores resultados no combate à sífilis.

Palavras-chave: Sífilis; Projeto de Resposta Rápida à Sífilis; Metodologia da problematização; Pesquisa-ação; Teoria do arco de Charles Maguerez.

\section{ABSTRACT}

This practical experience allowed to develop an action-reflection-action process of the care activities carried out by the health professionals from the Family Clinic, being an example of effective supporter's work, together with the territory's actors, aiming to produce changes in the management and care practices in advenced for better results in the fight against syphilis. The purpose of this article is to report the experience of conducting a workshop with health professionals, aiming at planning actions aimed at combating syphilis. Based on the action research methodology, a workshop was held at a Family Clinic located in a municipality in Baixada Fluminense/RJ. Problematization methodology was used, having Charles Maguerez's Theory of Arch as reference, analyzing the cyclical functioning in 5 steps: reality observation; definition of key points; theorizing; proposition of solution hypotheses; and, application to reality. Data were analyzed at each step and an experience report was produced. As a result, eight proposals were developed to achieve the aims of Rapid Response to Syphilis Project. This practical experience allowed to develop an action-reflection-action process of the care activities carried out by the health professionals from the Family Clinic, being an example of effective supporter's work, together with the territory's actors, aiming to produce changes in the management and care practices in advenced for better results in the fight against syphilis.

Key-words: Syphilis; Rapid Response to Syphilis Project; Problematization methodology; Action research; Charles Maguerez's Theory of Arch.

\section{INTRODUÇÃO}

A sífilis é considerada um grande problema de saúde pública, sendo decretada pelo Ministério da Saúde, em 2016, como uma epidemia. A taxa de detecção de sífilis adquirida em 2019 foi de 72,8/100.000 habitantes e da sífilis em gestante foi de 20,8/100.000 habitantes (BRASIL, 2020a). Já a incidência da sífilis congênita foi de 8,2/1.000 nascidos vivos para o mesmo período (BRASIL, 2020a). Os números apontam para a necessidade de controle dessa infecção e para a adoção de estratégias em prol do alcance da meta da Organização Mundial de Saúde (OMS) prevista entre os Objetivos de Desenvolvimento do Milênio (ODM), que é uma incidência de sífilis congênita de até 0,5/1.000 nascidos vivos (CHINAZZO, 2015).

Em 2017, foi aprovada a Lei Orçamentária Anual $N^{\circ} 13.414$, com previsão de recursos financeiros para utilização em ações de enfrentamento à sífilis (BRASIL, 2017). Tais recursos subsidiaram um acordo de cooperação técnica entre o Ministério da Saúde (MS), a Organização Pan-Americana (OPAS) e a Universidade Federal do Rio Grande do Norte (UFRN), resultando em um projeto intitulado "Resposta rápida ao enfrentamento 
da sífilis nas redes de atenção à saúde", também conhecido como Projeto "Sífilis não" (UFRN, 2017).

O objetivo geral do Projeto "Sífilis não" é contribuir para a redução da sífilis adquirida e em gestantes e a eliminação da sífilis congênita no Brasil (UFRN, 2017). Para isso, foram selecionados 52 apoiadores em todo o país para atuarem em 72 municípios. No estado do Rio de Janeiro, foram selecionados pelo projeto 13 municípios prioritários, escolhidos de acordo com o número de habitantes (mais de cem mil) e o índice composto de sífilis de cada município (LUCAS, 2019). Desses 13 municípios, 8 ficam localizados na região da Baixada Fluminense.

O apoio institucional é uma função gerencial para a cogestão que trabalha com as relações entre serviços, bem como entre gestores e trabalhadores. É um processo que movimenta um conjunto de agendas baseadas no tripé de envolvimentos coletivos, conexão de redes e junção de múltiplos olhares, práticas, interesses e desejos de um objetivo comum para implementação e fortalecimento de políticas públicas (LUCAS, 2019).

Visando ao trabalho nessas relações, durante uma reunião com o Grupo de Apoio Local (GAL), em um município da Baixada Fluminense/RJ, surgiu a ideia da realização de uma oficina com os profissionais das unidades de saúde de forma a fortalecer o diálogo horizontal entre gestores e profissionais, dividindo, assim, a corresponsabilização no combate à sífilis. A oficina seria uma integralização entre trabalhadores e gestores para a superação de uma situação-problema, influenciando positivamente nas práticas de atenção e de gestão com base no enfrentamento à sífilis, contribuindo, dessa forma, para alterar o grave quadro atual da sífilis no município em questão. Assim, o objetivo deste artigo é relatar a experiência da realização de uma oficina com a utilização da Teoria do Arco de Charles Maguerez realizada junto aos profissionais de saúde de uma Clínica da Família, localizada em um município da Baixada Fluminense/RJ.

\section{MÉTODO}

O presente relato de experiência utilizou como base a metodologia da pesquisa-ação, que é caracterizada por ser uma forma de pesquisa social empírica focada na relação, com uma ação voltada à resolução de um problema concreto, de caráter coletivo, no qual todos os envolvidos participam de forma ativa e cooperativa (THIOLLENT, 1986). A questão norteadora utilizada para estruturar a pesquisa foi: "Como podemos melhorar as práticas institucionais relacionadas à prevenção e ao controle da sífilis?".

Segundo Miranda e Resende (2006), a pesquisa-ação articula a teoria e a prática no processo de construção do conhecimento. Dessa forma, o trabalho sobre o público-alvo e as temáticas a ser trabalhadas (investigação) são convertidas em ações ao longo do processo, o que possibilita aos envolvidos uma atuação efetiva sobre a realidade estudada, visando transformá-la.

A pesquisa foi realizada em março de 2020, em uma Clínica da Família (CF), localizada em um município da Baixada Fluminense/RJ. Essa CF tem uma área de abrangência de 30 mil pessoas e é composta por 10 equipes de Saúde da Família, três equipes de Saúde Bucal e uma equipe do Núcleo de Apoio à Saúde da Família, oferecendo serviços de: curativos; planejamento familiar; teste do pezinho; testes rápidos de sífilis, HIV e hepatites; exames laboratoriais; consultas individuais e coletivas; pré-natal e puerpério, entre outros. A escolha por essa CF ocorreu em comum acordo com a gestão local, devido ao seu horário de funcionamento ser estendido, ao modelo integrado de assistência praticado e ao grande número de atendimentos realizados.

Foi realizada uma oficina com 24 profissionais da unidade de saúde, havendo pelo menos um profissional de cada equipe multiprofissional que atuava na CF. Essa amostra representativa foi fundamental para: i) compreender os aspectos facilitadores e limitantes de cada equipe durante 
o trabalho vivo em ato; ii) promover a troca de experiência entre os diversos atores da unidade; iii) propor alternativas de ação factíveis de execução, considerando as diversas realidades; iv) criar sensação de pertencimento, favorecendo a implementação da intervenção; e, v) favorecer o processo de disseminação das informações para os demais profissionais da unidade.

Durante a oficina, empregou-se a metodologia da problematização, tendo como referência a Teoria do Arco de Charles Maguerez. Trata-se de uma metodologia de ensino baseada na resolução de problemas, discutidos em grupo, voltada para o "saber fazer" a partir de uma realidade local, usando a experiência de cada um para a criação da solução do problema (BERBEL, 2020; REIBNNITZ, 2006). A estrutura do método envolve um funcionamento cíclico em cinco etapas: observação da realidade; definição dos pontos-chave; teorização; proposição de hipóteses de solução; e aplicação à realidade.

Para sistematizar a experiência, todos os dados e fatos vivenciados durante cada etapa da oficina foram registrados em um diário de campo. Todos os registros foram compartilhados com os participantes de forma a objetivar o que foi vivido e compreendido, incluindo: as referências dos acordos estabelecidos para funcionamento do grupo; os dados relacionados a compreensões, interpretações, sínteses das leituras de fundamentação teórica; descrição de atividades e práticas do grupo; e, sínteses das reflexões e decisões coletivas. Dessa forma, buscou-se construir os saberes coletivamente, num processo único, dialético e transformador dos participantes (FRANCO, 2005).

Os dados foram analisados em cada uma das etapas e foi produzido um relatório sobre a experiência. Foi estabelecido com a equipe um prazo de dois meses para avaliação das ações implementadas e identificação das mudanças necessárias para alcance dos objetivos do Projeto "Sífilis Não".

\section{RESULTADOS E LIÇÕES APRENDIDAS}

\section{A observação da realidade}

A "observação da realidade" é exercida com a participação ativa dos sujeitos, com um olhar sobre a realidade e os dados da vida real. Esse olhar permite aos sujeitos a identificação de aspectos que precisam ser trabalhados, desenvolvidos, revisados ou melhorados (BERBEL, 1999; BORDENAVE, 2004; BERBEL, 2020; OLIVEIRA, 2020).

Para auxiliar os participantes da oficina na análise da realidade, foram apresentados os dados de sífilis do Brasil, do estado do Rio de Janeiro e do município, disponíveis no Sistema Nacional de Agravos e Notificações (SINAN), do Departamento de Informática do Sistema Único de Saúde (Datasus). Além disso, buscamos nos sistemas de informações locais (SINAN) os dados de sífilis da CF em questão, e registros e documentos existentes na secretaria de saúde do município. Esses dados foram compostos por: número de casos de sífilis adquirida, sífilis em gestante e sífilis congênita. Verificou-se que, em alguns casos, a gestante havia realizado o pré-natal na CF, com resultado do VDRL (Venereal Disease Research Laboratory) indicativo de sífilis, porém, não havia sido notificada.

Além dos dados epidemiológicos, identificou-se o número de testes realizados, de preservativos distribuídos e de ações de educação em salas de espera realizadas pela unidade. Essa visão sobre os dados locais da CF teve por objetivo levar os profissionais da unidade a uma reflexão crítica sobre o seu processo de trabalho e o que essa "fotografia" nos dizia. Como lição aprendida, destaca-se a metodologia utilizada, pois, segundo a percepção dos participantes, contribuiu com um olhar sobre a realidade e como facilitadora para identificação das fragilidades. 


\section{Definição dos pontos-chave}

Na segunda etapa, definem-se o que é mais importante na realidade observada e quais são as variáveis para essa situação. Os participantes elegem os principais aspectos. É um momento de síntese em que os envolvidos suscitam os questionamentos que conduziram as discussões e reflexões sobre o tema em questão (BERBEL, 1999; BORDENAVE, 2004; BERBEL, 2020; OLIVEIRA, 2020).

Após a observação dos dados, iniciou-se uma discussão de quais os pontos centrais que precisariam ser alterados para a melhora daquela realidade. Foram identificados os possíveis fatores associados e determinantes do problema. Após reflexão, foram eleitos seis aspectos para ser estudados na etapa seguinte: 1. ausência/insuficiências de informações educativas sobre sífilis; 2. número de preservativos distribuídos; 3. necessidade de ampliação da oferta da testagem; 4. necessidade de realização de tratamento correto; 5. monitoramento das gestantes e parceiro; 6 . preenchimento correto da caderneta da gestante e das fichas de notificação.

A enumeração dos pontos-chave alinha-se às ações pensadas no Projeto "Sífilis Não", contemplando os eixos de cuidado integral, vigilância e educomunicação, mostrando uma relação conjunta entre os profissionais da assistência, gestores e apoiadora. Além disso, contribui para alcançar o objetivo geral do projeto que é contribuir para a redução da sífilis em gestantes, adquirida, e na eliminação da sífilis congênita no Brasil.

\section{Teorização}

$\mathrm{Na}$ aplicação da terceira etapa, instrumentalizamos os participantes com a apresentação do Protocolo Clínicos e Diretrizes Terapêuticas (PCDT) das Infecções Sexualmente Trasmitidas (IST) (BRASIL, 2020b) e o PCDT de Transmissão Vertical (BRASIL, 2019), além de buscas de subsídios acerca dos temas abordados. Nesse momento de teorização, todos os envolvidos devem estudar os assuntos. Deve-se eleger a forma de estudar cada ponto-chave. É a aquisição de um suporte teórico-científico que faça uma relação entre o empírico e a realidade. Uma boa teorização leva o sujeito a uma compreensão além do problema, incluindo os princípios teóricos que o justificam e explicam (BERBEL, 2020; OLIVEIRA, 2020).

Sobre o ponto-chave da ausência/insuficiências de informações educativas sobre sífilis, consultamos o site do Projeto "Sífilis Não" (https://sifilisnao.com.br/) para análise das peças informativas e educativas. Além disso, foram realizadas buscas de metodologias ativas que podem ser usadas em salas de esperas visando à responsabilização do sujeito sobre sua saúde.

Com relação à distribuição do preservativo, foram feitas buscas em artigos que falam sobre a distribuição e utilização de preservativos. Para embasar os pontos-chave de ampliação da oferta de testagem, realização de tratamento correto, monitoramento das gestantes e parceiros e preenchimento correto da caderneta da gestante, foram discutidos o PCDT de Infecções Sexualmente Transmissíveis e o PCDT de Prevenção da Transmissão Vertical de HIV, Sífilis e Hepatites Virais. Também realizamos uma revisão e reflexão da linha de cuidado da sífilis do município.

No decorrer dessa etapa, observou-se que alguns profissionais não tinham conhecimento da linha de cuidado sobre sífilis existente no município, que foi confeccionada pelos gestores e alguns profissionais. Também não tinham ciência da atualização do PCDT e das alterações mencionadas, assim como das peças de campanha sobre a sífilis disponíveis para utilização em campanhas educativas. Tratou-se, portanto, de importante momento de aprendizado e empoderamento dos profissionais presentes na oficina, com grande potencial de contribuição para o replanejamento das ações. 


\section{A proposição de hipóteses de solução}

A proposição de "hipóteses de solução" no Arco de Charles Maguerez visa elaborar alternativas viáveis para a solução dos problemas identificados nos pontos-chave, de forma criativa e crítica, a partir da relação teoria-prática (BERBEL, 2020; VIEIRA, 2015; OLIVEIRA, 2020). Na quarta etapa, os participantes pensaram e debateram as hipóteses apresentadas, avaliando a viabilidade e se a ação dependia de esforços internos do grupo.

Com relação à ausência/insuficiências de informações educativas sobre sífilis, as hipóteses propostas foram: incluir na televisão da sala de espera informações sobre sífilis; criar campanhas do "dia D" aos sábados com informações e testagens; e realizar registros das salas de espera dando ênfase sobre as informações sobre sífilis. A realização de campanhas e as informações na televisão na sala de espera também seriam alternativas para solucionar o problema com relação à distribuição dos preservativos. Para esse ponto-chave, também se sugeriu ampliar a oferta de preservativos durante as consultas, para além da disponibilização atual nos dispensadores. Com relação à ampliação da oferta da testagem, os profissionais propuseram realizar mais campanhas e oferecer o teste rápido de sífilis durante todas as consultas.

Visando ao tratamento correto, foi sugerido ampliar a divulgação dos esquemas terapêuticos previstos na linha de cuidado da sífilis para os profissionais da unidade e fomentar, junto aos médicos e enfermeiros, o processo de registro, na caderneta da gestante, sobre o tratamento de sífilis realizado. Esta última proposta também ajudaria a resolver o problema relacionado ao não preenchimento correto da caderneta da gestante e da notificação. Outra hipótese sobre esse ponto-chave referiu-se à realização de dupla checagem da caderneta por profissionais da CF, buscando diminuir 0 índice de incompletude das informações.
Diante da problemática relacionada ao monitoramento das gestantes e dos parceiros, a alternativa sugerida pelos profissionais foi a criação de uma planilha de monitoramento que será alimentada pela gestora da unidade e acompanhada pela coordenadora de Atenção Básica e superintendência de vigilância epidemiológica. Essa ferramenta teria informações sobre testagem, tratamento realizado e resultado de exames da gestante e do parceiro a fim de evidenciar os indivíduos que abandonaram o tratamento ou o realizaram de forma incorreta, favorecendo a atuação da equipe.

Percebe-se, com a execução dessa etapa, que as hipóteses geradas contemplam os pontos-chave evidenciados e todas estavam de acordo e subsidiadas pela teoria. Isso comprova que a metodologia utilizada favoreceu um repensar sobre o processo de trabalho, garantindo, assim, novos aprendizados.

\section{Aplicação à realidade}

Na quinta etapa, os sujeitos são levados a construir novos conhecimentos para transformar a realidade observada com base nas hipóteses levantadas. Há uma necessidade de refletir sobre a aplicabilidade à realidade encontrada de cada solução pensada (VIEIRA, 2015; OLIVEIRA, 2020).

O grupo analisou as hipóteses de solução de acordo com a exequibilidade, a urgência e a prioridade e verificou que todas as hipóteses eram passíveis de aplicabilidade e optaram por implementar as ações planejadas. As ações propostas foram: i) informações sobre sífilis com a divulgação das peças de comunicação do projeto nas salas de espera; ii) campanha sobre sífilis com informações, testagem e oferta de preservativos; iii) realização de sala de espera abordando a temática da sífilis; iv) ampliação da oferta do preservativo; v) ampliação da oferta de testagem; vi) divulgação do esquema terapêutico junto aos médicos e enfermeiros; vii) anotação na caderneta da gestante com 
conferência de outro profissional, bem como preenchimento correto da notificação; viii) implantação da planilha de monitoramento da gestante e parceiros, compartilhada entre profissionais e gestores, com informações sobre testagem, tratamento realizado e resultado de exames.

Ao finalizarmos o encontro, apresentamos - planejamento das ações, que foi construído pelo grupo, com base no exercício ação-reflexão-ação e a contribuição da apoiadora como facilitadora na transformação da realidade local. Foram grandes as lições de aprendizado não só como uma ferramenta de gestão mas principalmente pelo envolvimento do profissional, contribuindo para a construção de uma sensação de pertencimento e responsabilização e para o fortalecimento da colaboração coletiva, da autonomia e do empoderamento.

Quadro 1 - Sistematização do Arco da Problematização de Charles Maguerez da oficina realizada numa Clínica da Família de um município da Baixa Fluminense.

\begin{tabular}{|c|c|c|c|c|}
\hline $\begin{array}{l}\text { Observação } \\
\text { da realidade }\end{array}$ & Pontos-chave & Teorização & $\begin{array}{l}\text { Hipóteses de } \\
\text { solução }\end{array}$ & $\begin{array}{l}\text { Aplicação da } \\
\text { realidade (prazo } \\
2 \text { meses) }\end{array}$ \\
\hline \multirow[t]{2}{*}{$\begin{array}{l}\text { Alta } \\
\text { incidência de } \\
\text { casos de sífilis } \\
\text { no município } \\
\text { e na Unidade } \\
\text { de Saúde. } \bigcirc \\
\text { que fazer } \\
\text { para reduzir? }\end{array}$} & $\begin{array}{l}\text { Ausência/ } \\
\text { insuficiências de } \\
\text { informações educativas } \\
\text { sobre Sífilis }\end{array}$ & $\begin{array}{l}\text { Mostra das peças de } \\
\text { campanhas disponíveis no } \\
\text { site do Projeto "Sífilis Não"; } \\
\text { Artigos que comprovem } \\
\text { a importância de } \\
\text { ações educativas com } \\
\text { metodologias ativas. }\end{array}$ & $\begin{array}{l}\text { Incluir na TV da } \\
\text { sala de espera } \\
\text { informações sobre } \\
\text { a sífilis; } \\
\text { Criar campanhas do } \\
\text { dia D aos sábados } \\
\text { com informações e } \\
\text { testagem; } \\
\text { Realizar registros } \\
\text { das salas de espera } \\
\text { e dar ênfase às } \\
\text { informações } \\
\text { sobre Sífilis. }\end{array}$ & $\begin{array}{l}\text { TV na sala de espera } \\
\text { com informações } \\
\text { sobre sífilis. } \\
\text { Campanhas com } \\
\text { oferta de testagem, } \\
\text { informação e } \\
\text { preservativo; } \\
\text { Sala de espera } \\
\text { abordando a sífilis; }\end{array}$ \\
\hline & $\begin{array}{l}\text { Distribuição de } \\
\text { preservativos }\end{array}$ & $\begin{array}{l}\text { Estudos sobre o uso de } \\
\text { preservativos; }\end{array}$ & $\begin{array}{l}\text { Incluir na TV da } \\
\text { sala de espera } \\
\text { informações sobre o } \\
\text { uso do preservativo } \\
\text { e disponibilidade na } \\
\text { unidade; } \\
\text { Incluir na TV da } \\
\text { sala de espera } \\
\text { informações sobre o } \\
\text { uso do preservativo } \\
\text { e disponibilidade na } \\
\text { unidade; } \\
\text { Ofertar também nas } \\
\text { consultas, além dos } \\
\text { preservativos que } \\
\text { já estão disponíveis } \\
\text { nos dispensadores. } \\
\text { Criar campanhas do } \\
\text { dia D aos sábados } \\
\text { com informações, } \\
\text { testagem e ofertas } \\
\text { de preservativos. }\end{array}$ & $\begin{array}{l}\text { TV na sala de espera } \\
\text { com informações } \\
\text { sobre sífilis. } \\
\text { Campanhas com } \\
\text { oferta de testagem, } \\
\text { informação e } \\
\text { preservativo; } \\
\text { Ampliar oferta de } \\
\text { preservativo. }\end{array}$ \\
\hline
\end{tabular}




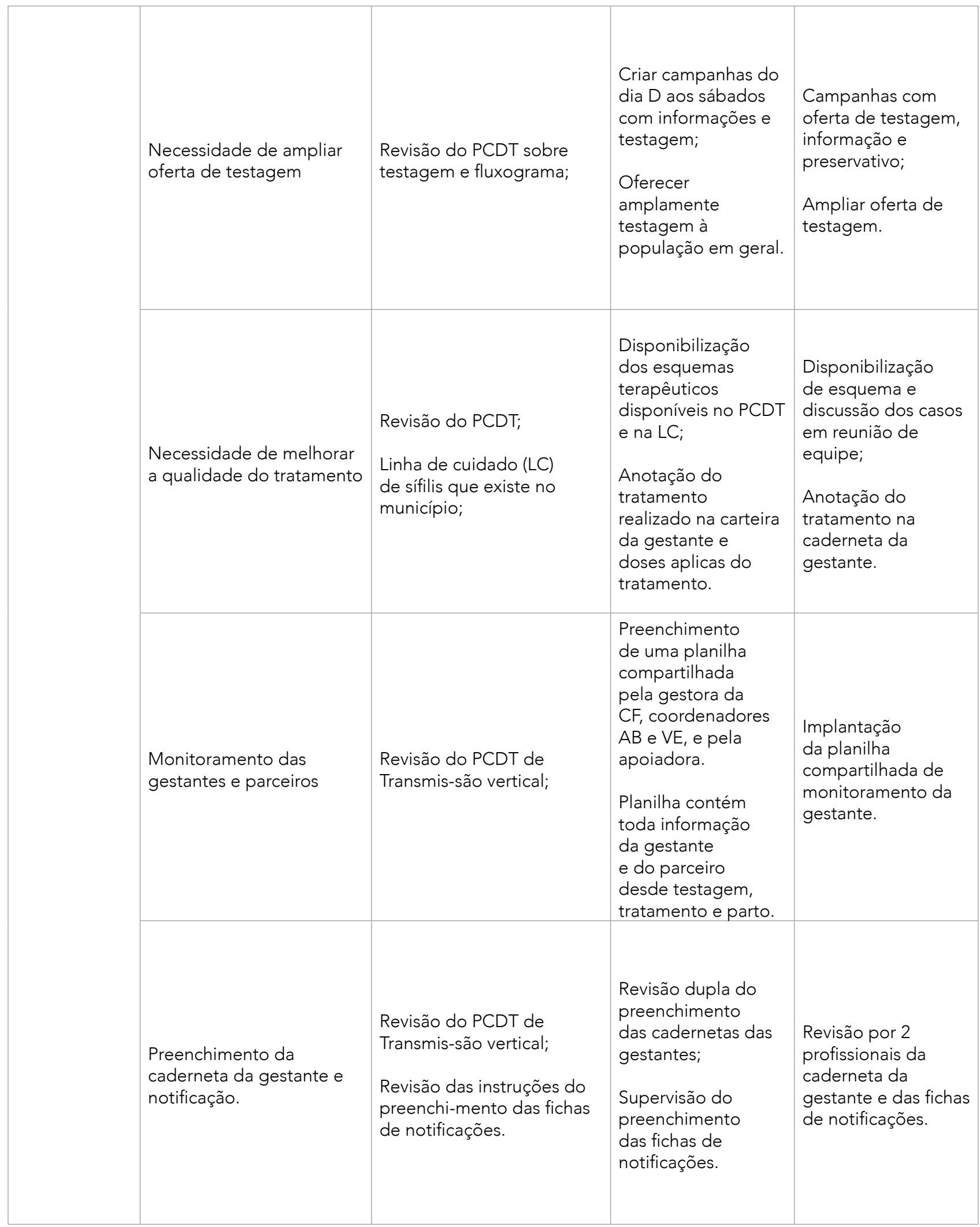

Fonte: autoria própria.

\section{CONSIDERAÇÕES FINAIS}

A aplicação da metodologia do Arco de Charles Maguerez contribuiu para fortalecer o papel dos profissionais de saúde no planejamento das ações do projeto e como agente transformador. Ao olhar para a realidade e para os dados produzidos na sua unidade, foi possível observar o que era necessário ajustar visando à melhora dos 
indicadores. A etapa de teorização forneceu subsídios que permitiram aos profissionais pensar em ações práticas que pudessem transformar a realidade encontrada, permitindo a elaboração de um plano de ação para a unidade, construído de forma interdisciplinar pelos profissionais envolvidos.

Durante a oficina, foi percebido um relevante processo de reorientação das práticas de gestão e do serviço de saúde, por meio de uma relação horizontal pautada pela corresponsabilização, diminuindo a fragmentação e a dificuldade de acesso dos usuários aos serviços de saúde e às ações, sendo essa uma das atribuições do apoio institucional. Essa oficina contribuiu, ainda, para instrumentalizar os profissionais para o uso de uma metodologia que pode ser aplicada em outros momentos para o realinhamento das ações de combate à sífilis, ou, ainda, para o enfrentamento de outros agravos de saúde e revisão de processos de trabalho.

Ressalta-se que alguns desafios precisam ser superados, tais como: a realização dessa oficina nas outras Clínicas da Família do município e o retorno para o monitoramento da atividade. As ações tinham o prazo imediato para implantação/implementação, sendo prevista uma segunda oficina em dois meses para monitoramento das ações e análise dos problemas inicialmente detectados. Porém, devido à pandemia da covid-19, ainda não foi possível a realização dessa etapa.

Apesar disso, os produtos da oficina foram encaminhados para o Comitê de Transmissão Vertical Municipal e ao Grupo de Apoio Local, que estão se articulando em prol da continuidade das atividades. A execução dessa oficina é um exemplo de trabalho efetivo do apoio em conjunto com os atores do território a fim de produzir mudanças nas práticas de gestão e assistência na busca de melhores resultados no combate à sífilis.

\section{AGRADECIMENTOS}

Ao Projeto "Sífilis Não", pela oportunidade de participar e por todo o aprendizado compartilhado. Aos participantes da oficina, pela disponibilidade, pelo respeito, pela responsabilidade e pelo compromisso. Ao Secretário de Saúde, ao Superintendente de Vigilância Epidemiológica, à Coordenadora da Atenção Básica, ao consultor em saúde, ao Coordenador de IST e ao Coordenador de dados vitais, pela confiança e pelo apoio para execução do projeto e realização da oficina. 


\section{REFERÊNCIAS}

BERBEL, N. A. N. Metodologia da problematização: fundamentos e aplicações. Londrina: INP/UEL, 1999.

BERBEL, N. A. N. As metodologias ativas e a promoção da autonomia de estudantes.

Semina: Ciências Sociais e Humanas, Londrina, v.32, n.1, p. 25-40, jan./jun. 2011. Disponível em: http://www.uel.br/revistas/uel/index.php/seminasoc/article/view/10326. Acesso em: 28 out. 2020

BORDENAVE, J. D.; PEREIRA, A. M. P. Estratégias de ensino-aprendizagem. 25. ed. Rio de Janeiro: Vozes, 2004.

BRASIL. Lei $\mathbf{n} .^{\circ}$ 13.414, de 10 de janeiro de 2017. Estima a receita e fica a despesa da União para o exercício financeiro de 2017. Disponível em: http://www.planalto.gov.br/ ccivil_03/_Ato2015-2018/2017/Lei/L13414.htm\#: :text=Estima\%20a\%20receita\%20e\%20 fixa,cargo\%20de\%20PRESIDENTE\%20DA\%20REP\%C3\%9ABLICA. Acesso em: 15 mar. 2021.

BRASIL. Ministério da Saúde. Protocolo Clínico e Diretrizes Terapêuticas para Prevenção da Transmissão Vertical do HIV, Sífilis e Hepatites Virais. Brasília: Ministério da Saúde, 2019.

BRASIL. Ministério da Saúde. Boletim Epidemiológico de Sífilis, Brasília, ano VI, n. 1, p. 1-43, 2020a.

BRASIL. Ministério da Saúde. Protocolo Clínico e Diretrizes Terapêuticas para Atenção Integral às Pessoas com Infecções Sexualmente Transmissíveis (IST). Brasília: Ministério da Saúde, 2020b.

CHINAZZO, L. K.; DE LEON, C. A. Perfil clínico e epidemiológico da sífilis congênita na unidade de internação de um hospital universitário. Boletim Científico de Pediatria, v.4, n. 3, p.65-69, 2015.

FRANCO, M. A. S. Pedagogia da pesquisa-ação. Educação e Pesquisa, v. 31, n. 3, p. 483502, 2005.

LUCAS, M. C. V. et al. A experiência do apoio institucional do projeto de resposta rápida à sífilis nas redes de atenção à saúde. Revista Brasileira de Inovação Tecnológica em Saúde, v. 9, n. 2, p.8-24, 2019. Edição especial - Projeto Sífilis Não. Disponível em: https:// periodicos.ufrn.br/reb/article/view/18679. Acesso em: 28 out. 2020.

MIRANDA, M. G.; RESENDE, A. C. A. Sobre a pesquisa-ação na educação e as armadilhas do praticismo. Revista Brasileira de Educação, v. 11, n. 33, p.511-565, set./dez. 2006.

OLIVEIRA, J. F. S.; VELOSO, D. L. C.; OLIVEIRA, S. L. F. Arco de Maguerez: a gamificação como ferramenta educativa no cuidado ao pé diabético. Revista Brasileira de Educação e Saúde, v. 10, n. 1, p.95-99, jan./mar. 2020. 
REIBNITZ, K. S.; PRADO, M. L. Inovação e Educação em Enfermagem. Florianópolis:

Cidade Futura, 2006.

THIOLLENT, M. Metodologia da pesquisa-ação. 2. ed. São Paulo: Cortez, 1986.

UNIVERSIDADE FEDERAL DO RIO GRANDE DO NORTE (UFRN). Plano de Trabalho do Projeto de Pesquisa aplicada para a integração inteligente orientada ao fortalecimento das redes de atenção para resposta rápida à sífilis. Natal: UFRN, 2017. 65p.

VIEIRA, M. N. C. M.; PANUNCIO-PINTO, M. P. A Metodologia da Problematização (MP) como estratégia de integração ensino-serviço em cursos de graduação na área da saúde. Medicina, Ribeirão Preto, v. 48, n. 3, p. 241-248, 2015. Disponível em: http://www.revistas. usp.br/rmrp/article/view/104310. Acesso em: 28 out. 2020. 